\title{
Cloning and nucleotide sequencing of a 15 kb region of the Bacillus subtilis genome containing the iol operon
}

\author{
K. Yoshida, ${ }^{1}$ H. Sano, ${ }^{1}$ Y. Miwa, ${ }^{1}$ N. Ogasawara ${ }^{2}$ and $Y$. Fujita $^{1}$ \\ Author for correspondence: Y. Fujita. Tel: +8184936 2111. Fax: +81849362023.
}

1 Department of
Biotechnology, Faculty of
Engineering, Fukuyama
University, Fukuyama 729-
02 , Japan
2 Advanced Institute of
Science and Technology,
Nara, Ikoma 630-01, Japan

Within the framework of an international project on the sequencing of the whole Bacillus subtilis genome, a $15 \mathrm{~kb}$ chromosome segment, which contains the iol operon involved in inositol utilization, has been cloned and sequenced. This region (14974 bp) contains 12 complete open reading frames (ORFs; genes) and two partial ones; the seventh gene (E83G) is the idh gene encoding inositol dehydrogenase. All the genes identified are transcribed in the same direction as that of the movement of the replication fork. A homology search for their products deduced from the 12 complete ORFs revealed that eight of them exhibit significant homology to known proteins such as fructokinase, acetolactate synthase, fructose-1,6-bisphosphate aldolase (B. subtilis), and PhoB and FtsE proteins (Escherichia coli). It also implied that two genes (B65D and B65E) might encode a set of two-component regulatory proteins and that the B65F gene might encode a protein belonging to the ATP-binding cassette (ABC) family. Based on the features of the nucleotide sequence determined and the results of the homology search, the primary structure of the iol operon is predicted.

Keywords: Bacillus subtilis, genomic sequencing, inositol operon, OmpR subfamily, ABC family

\section{INTRODUCTION}

Recent developments of new techniques for DNA sequencing and analysis, including improvement of DNA sequencers and microcomputer software, allows us to sequence the whole genome of an organism. But even now, a vast amount of work is required to determine the entire nucleotide sequence of a relatively small bacterial genome, so it is important to develop a productive and systematic strategy. The systematic sequencing of a small genome will provide much productive information and allow the technical developments required to determine the entire sequence of the large genomes of higher eukaryotes.

Recently, several sequencing projects on small genomes have been started. Among them, the Bacillus subtilis

Abbreviations : aa, amino acid; ABC, ATP-binding cassette; GP, translated protein sequence from NCBI-GenBank Nucleotide Sequence Database; SD, Shine-Dalgarno; SP, Swiss Prot Protein Sequence Database.

The EMBUGenBank/DDBJ accession number for the nucleotide sequence (14974 bp) reported in this paper is D14399. sequencing project is an international cooperative effort between Japan and Europe, the aim of which is to complete the entire sequence within the next five years. We are participating in this project, and are responsible for the sequencing of an approximately $180 \mathrm{~kb}$ chromosomal region between $\operatorname{sac} S\left(333^{\circ}\right)$ and gnt $\left(344^{\circ}\right)$, in which the iol operon involved in myo-inositol utilization is located (Fujita \& Fujita, 1983).

In this first communication, we describe our sequencing strategy for this project and report the determination of the nucleotide sequence of a $14974 \mathrm{bp}$ region containing the $i d b$ gene (Fujita et al., 1991) which belongs to the iol operon.

\section{METHODS}

Bacterial strains, phages, plasmids and growth media. $B$. subtilis strain $1 \mathrm{~A} 1$ (Marburg 168; $\operatorname{trp} C 2$ ) was chosen as our target strain. Escherichia coli strains P2392, a P2 lysogen of strain LE392 [bsdR 514( $\left.\mathrm{r}_{\mathrm{k}}^{-} \mathrm{m}_{\mathrm{k}}^{+}\right)$supE44 supF58 lac Y or $\Delta($ lacIZY) 6 galT22 galK2 metB1 trpR 55 mor $A^{-}$morB $B^{+}$, and XL1-Blue \{end $A 1$ bsdR17 $\left(\mathrm{r}_{\mathrm{k}}^{-} \quad \mathrm{m}_{\mathrm{k}}^{+}\right)$supE44 thi-1 $\operatorname{rec} A 1$ gyrA96 relA1 $\Delta($ lac $)$ $\mathrm{F}^{\prime}\left[\right.$ pro $A B^{+}$lacl $I^{\mathrm{q}}$ lac $\left.\left.Z \Delta \mathrm{M} 15 \operatorname{Tn} 10 \mathrm{Tet}^{\mathrm{r}}\right]\right\}$ were used as cloning hosts for $\lambda$ DASH II and M13mp19 phage clones, respectively. 


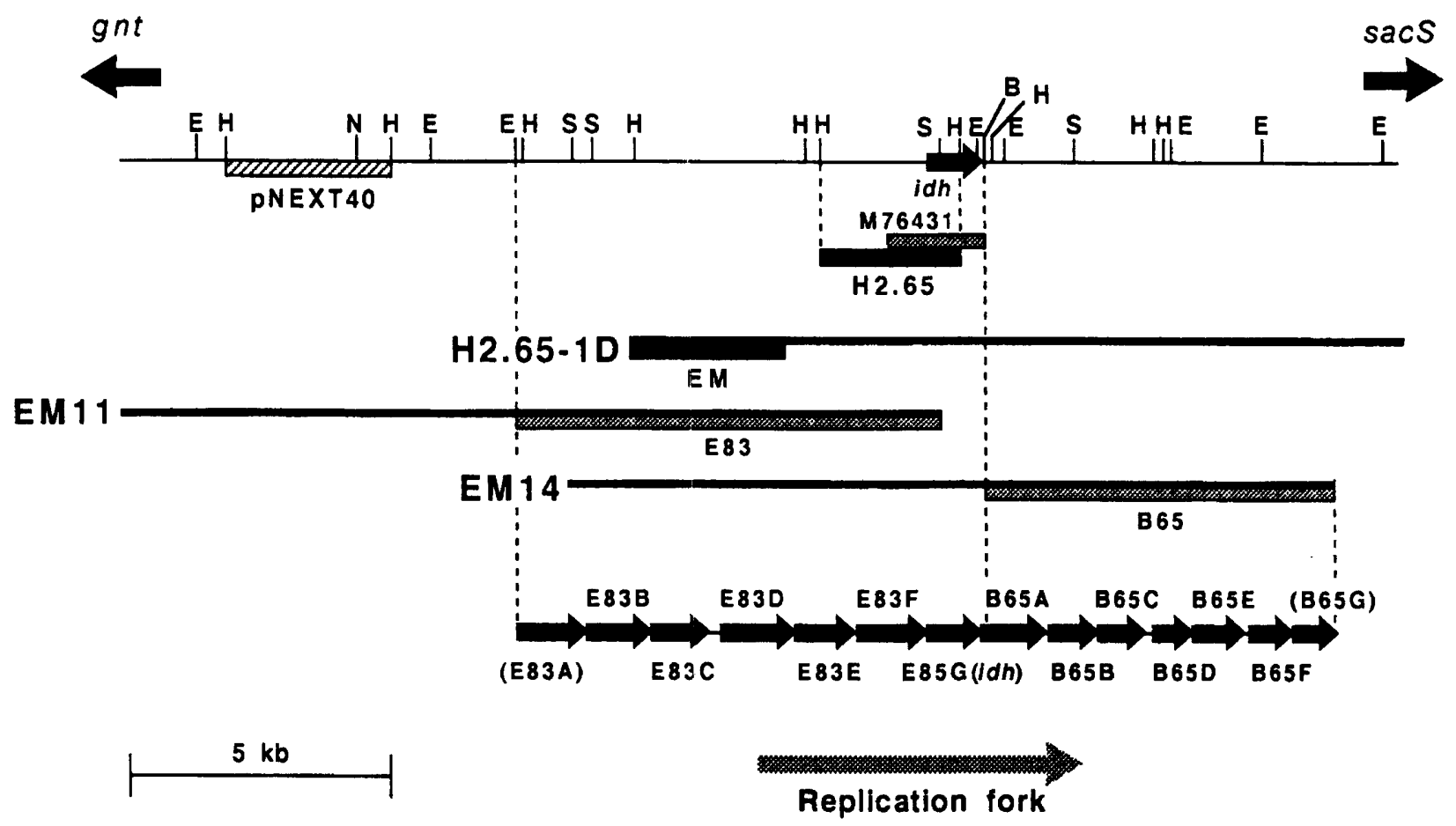

Fig. 1. Cloning, physical mapping and sequencing of the iol region. $\lambda$ DASH II clones (H2.65-1D and EM11 and 14), the inserts of which are indicated by thick solid lines, were obtained using DNA fragments as probes (solid boxes; $\mathrm{H} 2.65$ and $E M)$. A physical map of the cloned $24 \mathrm{~kb}$ region with respect to EcoRI (E), BamHI (B), HindIII (H), Sall (S) and Notl (N) is shown, in which the idh gene and the insert of plasmid pNEXT40 (Itaya \& Tanaka, 1991) are located. This map was constructed by connecting the physical maps of the inserts of the three $\lambda$ DASH II clones, which were obtained as described in Methods. The direction of the location of gnt and sacS is indicated at the top of the figure. The sequenced regions are indicated as stippled boxes [M76431, accession no. in the EMBL/GenBank/DDB] Nucleotide Sequence Database (Fujita et al, 1991); E83 and B65]. In the lower part of the figure, the deduced 14 ORFs in the connected sequenced region (14974 bp) are shown; the partial ORFs are showr in parentheses. The direction of movement of the replication fork is indicated at the bottom of the figure.

Strain 1A1, strains P2392, XL1-Blue and $\lambda$ DASH II, and M13mp19 were obtained from the Bacillus Genetic Stock Center (Ohio) via M. Itaya (Mitsubishi Kasei Institute of Life Sciences, Tokyo), Stratagene (La Jolla), and Takara Shuzo (Kyoto), respectively.

Plasmids pIOL02 and pNEXT40 were used for preparation of DNA probes for hybridization. pIOL02 carries the idh gene (Fujita et al., 1991). pNEXT40 is one of the NotI linking clones located at $4082 \mathrm{~kb}$ on the B. subtilis chromosome (Itaya \& Tanaka, 1991); it was supplied by M. Itaya

$\mathrm{NZY}$ broth $\left[5 \mathrm{~g} \mathrm{NaCl} \mathrm{l}^{-1}, 2 \mathrm{~g} \mathrm{MgSO}{ }_{4} \cdot \mathrm{H}_{2} \mathrm{Ol}^{-1}, 5 \mathrm{~g}\right.$ yeast extract $\mathrm{I}^{-1}$ and $10 \mathrm{~g} \mathrm{NZ}$ amine (Wako, Osaka) $\left.1^{-1} ; \mathrm{pH} 7 \cdot 5\right]$ and L-broth (10 $\mathrm{g}$ tryptone $\mathrm{l}^{-1}, 5 \mathrm{~g}$ yeast extract $\mathrm{l}^{-1}$ and $5 \mathrm{~g} \mathrm{NaCl} \mathrm{l}^{-1}$ ) were used for growth of the E. coli strains to propagate $\lambda$ DASH II and $\mathrm{M} 13 \mathrm{mp} 19$ phage clones, respectively.

Preparation of a B. subtilis genomic library. A B. subtilis genomic library using $\lambda$ DASH II was prepared according to the instruction manual from Stratagene. The chromosomal DNA of $B$. subtilis strain $1 \mathrm{~A} 1$ was isolated and partially digested with Sau $3 \mathrm{AI}$ to yield DNA fragments of approximately $15 \mathrm{~kb}$. Fragments ranging from 10 to $20 \mathrm{~kb}$ were fractionated by agarose gel electrophoresis, dephosphorylated with calf intestinal alkaline phosphatase (Boehringer Mannheim), and ligated to the arms of $\lambda$ DASH II cleaved with BamHI. The ligation mixture was incubated with an in vitro packaging mixture (GIGAPACK II XL, Stratagene), and the resulting phage particles were propagated on strain P2392 for Spi/P2 selection. Nineteen thousand independent phage clones, from several packaging experiments, were amplified by plaque formation to yield a $B$. subtilis genomic library $\left(2.5 \times 10^{7}\right.$ p.f.u. $\left.\mathrm{ml}^{-1}\right)$.

Isolation of $\lambda$ DASH II clones containing a DNA insert carrying the iol locus and construction of a physical map of the DNA inserts. Using an aliquot of the $B$. subtilis genomic library, approximately 12000 plaques were formed in total and subjected to plaque hybridization (Benton \& Davis, 1977) with an appropriate DNA fragment as probe. EcoRI digests of phage DNA prepared from positive recombinant clones were subjected to agarose gel electrophoresis and Southern hybridization (Southern, 1975) to confirm that the DNA inserts actually carried the iol locus. A physical map of the region cloned in $\lambda$ DASH II was constructed according to the method described by Kohara et al. (1987), as follows. Recombinant phage DNA was partially digested with EcoRI, HindIII, SalI, Bam HI or NotI, and subjected to gel electrophoresis using $0.4 \%(\mathrm{w} / \mathrm{v})$ agarose $\left(0.7 \mathrm{~V} \mathrm{~cm}^{-1}, 40 \mathrm{~h}\right)$, and Southern hybridization using the $9 \mathrm{~kb}$ arm of $\lambda$ DASH II as probe.

Shotgun cloning for random sequencing. $\lambda$ DASH II recombinant phage DNA carrying the iol locus was isolated, 
digested with an appropriate restriction enzyme, and approximately $5 \mu \mathrm{g}$ of the DNA fragment to be sequenced $(5-10 \mathrm{~kb}$ in length) was purified. This DNA fragment was self-ligated and partially digested with DNaseI (Takara Shuzo) in the presence of $\mathrm{Mn}^{2+}$ to generate random fragments $400-1000$ bp in length. The resulting fragments were blunt-ended by stepwise treatment with T4 DNA polymerase and the Klenow fragment of DNA polvmerase I. The blunt-ended fragments were ligated with M13mp19 replicative-form DNA, which had been digested with Smal, extensively dephosphorylated with calf intestine alkaline phosphatase, and transferred to competent cells of strain XI.1-Blue for the screening of white plaques on plates $[\mathrm{H}-$ broth (10 $\mathrm{g}$ tryptone $\mathrm{l}^{-1}$ and $\left.\left.5 \mathrm{~g} \mathrm{NaCl}^{-1}\right)\right]$ containing 5-bromo4-chloro-3-indolyl $\beta$-D-galactoside and isopropyl $\beta$-Dthiogalactopyranoside. Hundreds of white plaques were randomly selected, picked up with toothpicks, and phage particles were suspended in $50 \mu \mathrm{L}$-broth and incubated at $37^{\circ} \mathrm{C}$ for $5 \mathrm{~h}$ in 96 -well microtitre plates to propagate the recombinant phage clones. The insert DNA of each recombinant M13 phage was amplified by 30 cycles of PCR $\left(20 \mathrm{~s}\right.$ at $95^{\circ} \mathrm{C}, 20 \mathrm{~s}$ at $50^{\circ} \mathrm{C}$ and $1 \mathrm{~min}$ at $72^{\circ} \mathrm{C}$ ) with Vent DNA polymerase (New England Biolabs), using a pair of primers, M4 and RV ( $1 \mu \mathrm{M}$ each; Takara Shuzo), and the propagated-phage suspensions directly as template solutions with a Perkin Elmer GeneAmp PCR System 9600 (Takara Shuzo). The amplified products were analysed by agarose gel electrophoresis, and the recombinant phages carrying insert DNA ranging from 400 to $1000 \mathrm{bp}$ were selected for preparation of templates in subsequent DNA sequencing reactions.

DNA sequencing. Sequencing reactions were performed using a Dye-Primer Cycle Sequencing Kit with -21 M13 Dye Primer (Applied Biosystems). Template DNAs were insert DNAs of the recombinant $\mathrm{M} 13$ phages selected, which were amplified by 30 cycles of PCR $\left(30 \mathrm{~s}\right.$ at $95^{\circ} \mathrm{C}, 30 \mathrm{~s}$ at $50^{\circ} \mathrm{C}$ and $2 \mathrm{~min}$ at $\left.72^{\circ} \mathrm{C}\right)$, with AmpliTaq DNA polymerase (Perkin-Elmer Cetus), using M4 and RV (0.05 $\mu \mathrm{M}$ each) as primers with a Program Temp Control System PC-700 (Astec, Fukuoka), and purified by precipitation with polyethylene glycol. The reaction products were applied to and sequenced with a DNA sequencer (373A, Applied Biosystems). Sequences determined randomly were compiled and automatically connected using a personal computer with the GENETYX-MAC 6.0.1 and GENETYX-MAC/ATSQ programs (Software Development Co., Tokyo), respectively; to sequence a $10 \mathrm{~kb}$ fragment as a target, for example, 150 independent sequences on average were needed to form several islands of connected sequences. The insert DNAs of M13 recombinant clones located at the extremities of each of these islands were sequenced with an anti-sense primer (M13 Reverse Dye Primer, Applied Biosystems). Sequences newly and previously determined were all automatically connected to shorten the gaps between the islands. The islands were physically aligned on a constructed physical map of the target DNA fragment by Southern hybridization using the DNA inserts belonging to different islands as probes. The gap regions neighbouring islands were amplified by PCR, using B. subtilis chromosomal DNA as a template, and synthetic primer pairs each consisting of an approximately 20 base sequence complementary to one of the extremities of a neighbouring island and the same nucleotide sequence as one of each set of different dyeprimers (18-20 bases). New sequences were obtained using the PCR products as templates, which were automatically connected with all those previously determined. The last process was repeated until sequencing of the target fragment was completed.

Computer analysis. The DNA sequences determined were analysed with the GENETYX-MAC 6.0 .1 program. A homology search for putative gene products was performed with the FASTA program (Pearson \& Lipman, 1988) using an NEC PC-9801 personal computer with GENETYX-CD Version 22.0.0 (Software Development Co.) and a GenomeNet FASTA server (Supercomputer Laboratory, Institute for Chemical Research, Kyoto University) (e-mail: fasta@genome.ad.jp).

\section{RESULTS AND DISCUSSION}

\section{Cloning, physical mapping and sequencing of the iol region}

Screening of the B. subtilis genomic library constructed using $\lambda$ DASH II was started with a HindIII $2.65 \mathrm{~kb}$ fragment (H2.65) derived from plasmid pIOL02 (Fujita $e t$ al., 1991) as a probe (Fig. 1), which contained the $i d h$ gene, the nucleotide sequence of which was reported previously (Fujita et al., 1991). With this probe, a $\lambda$ DASH II recombinant clone, H2.65-1D, carrying the HindIII $2.65 \mathrm{~kb}$ fragment was isolated. Using a $3 \mathrm{~kb}$ EcoRI-MluI fragment (EM) derived from DNA of recombinant clone H2.65-1D as another probe (Fig. 1), $\lambda$ DASH II clones EM11 and EM14 were obtained. Fig. 1 also shows the physical map of the $24 \mathrm{~kb}$ region containing the $i d b$ gene with respect to EcoRI, BamHI, HindIII, SalI and NotI. This map was constructed by connecting those of the insert DNAs of the three recombinant clones, produced by the method of Kohara et al. (1987). The HindIII insert in plasmid pNEXT40, which is one of the NotI linking clones located at $4082 \mathrm{~kb}$ on the B. subtilis chromosome (Itaya \& Tanaka, 1991), hybridized with the insert of the EM11 clone (data not shown), suggesting that the NotI location of the EM11 insert DNA is at $4082 \mathrm{~kb}$. The idh

\section{Table 1. General features of ORFs found in the $14974 \mathrm{bp}$ iol region sequenced}

\begin{tabular}{|c|c|c|c|c|}
\hline ORF & \multicolumn{2}{|c|}{ Endpoints (bp) } & $\begin{array}{l}\text { Size } \\
\text { (aa) }\end{array}$ & Translation start* \\
\hline E83A† & 1 & 1253 & & \\
\hline$E 83 B$ & 1331 & 2143 & 271 & gAGGAGGgcttctctatg \\
\hline$E 83 C$ & 2170 & 3144 & 325 & gAGGAGtgGctgtttacgatg \\
\hline$E 83 D$ & 3351 & 5090 & 580 & cAGGAtGcaggccatttg \\
\hline$E 83 E$ & 5110 & 6000 & 297 & ggGGAGGcaccatagatg \\
\hline$E 83 F$ & 6018 & 7334 & 439 & tgGGgaaTGaacatatg \\
\hline$E 83 G(i d b)$ & 7360 & 8391 & 344 & AAGGAGtgGctgtcaatg \\
\hline$B 65 A$ & 8414 & 9280 & 289 & gAGGAGtgaaaatatg \\
\hline$B 65 B$ & 9369 & 10202 & 278 & AtGGgGGaGtctggcatg \\
\hline$B 65 C$ & 10226 & 11095 & 290 & AAGGAGGgGtgaatatg \\
\hline$B 65 D$ & 11207 & 11893 & 229 & cgGGAGtgGttcagtttg \\
\hline$B 65 E$ & 11893 & 12867 & 325 & gctGAGGgcgcagtcatg \\
\hline$B 65 F$ & 13014 & 13784 & 257 & AAGGAGGattttcatg \\
\hline$B 65 G \dagger$ & 13762 & 14974 & & ggGGAaacGcaaatg \\
\hline
\end{tabular}

* Putative initiation codons are shown in bold face. The same nucleotides as those of a consensus SD sequence of AAGGAGGTG are shown in upper case.

tPartial ORF. 
Table 2. Results of comparison of eight ORFs with protein databases

\begin{tabular}{|c|c|c|c|}
\hline ORF* & Homologous protein & Database: entry $\nmid$ & $\begin{array}{l}\text { FASTA } \\
\text { optimized } \\
\text { score }\end{array}$ \\
\hline$E 83 C$ & $\begin{array}{l}\text { Fructokinase } \\
\text { (Solanum tuberosum) } \\
\text { (Vibrio alginolyticus) } \\
\text { (Klebsiella pneumoniae) } \\
\text { (Salmonella typhimurium) }\end{array}$ & $\begin{array}{l}\text { GP:STFRUCM_1 } \\
\text { PIR:jQ0782 } \\
\text { PIR:s18523 } \\
\text { PIR:s18524 }\end{array}$ & $\begin{array}{l}312 \\
243 \\
237 \\
235\end{array}$ \\
\hline$E 83 D$ & $\begin{array}{l}\text { Acetolactate synthase } \\
\text { (Saccharomyces cerevisiae) } \\
\text { (Spirulina platensis) } \\
\text { (Lactococcus lactis) } \\
\text { (Bacillus subtilis) } \\
\text { Acetohydroxy acid synthase } \\
\text { (Corynebacterium glutamicum) } \\
\text { Pyruvate dehydrogenase } \\
\text { (Escherichia colt) }\end{array}$ & $\begin{array}{l}\text { PIR: } a 23808 \\
\text { PIR: b44857 } \\
\text { SP:ILVB_LACLA } \\
\text { GP:BACILNB_1 }\end{array}$ & $\begin{array}{l}334 \\
302 \\
298 \\
295\end{array}$ \\
\hline$E 83 F$ & $\begin{array}{l}\text { Quinolone resistant protein NorA } \\
\text { (Stapbylococcus aureus) } \\
\text { Methylenomycin A resistant protein MMR } \\
\text { (Bacillus subtilis) } \\
\text { D1 transporter } \\
\text { (Leishmania donovani) } \\
\text { Arabinose transporter } \\
\text { (Escherichia coli) }\end{array}$ & $\begin{array}{l}\text { PIR : a } 37838 \\
\text { PIR : } 222742 \\
\text { GP:LEID1TRA_1 } \\
\text { PIR : a28075 }\end{array}$ & $\begin{array}{l}235 \\
175\end{array}$ \\
\hline$E 83 G(i d h)$ & $\begin{array}{l}\text { Streptomycin biosynthesis protein StrI } \\
\text { (Streptomyces griseus) }\end{array}$ & PIR: s17779 & 285 \\
\hline$B 65 C$ & $\begin{array}{l}\text { Tsr protein } \\
\text { (Bacillus subtilis) } \\
\text { Fructose bisphosphate aldolase I } \\
\text { (Rbodobacter sphaeroides) } \\
\text { Fructose bisphosphate aldolase II } \\
\text { (Rbodobacter sphaeroides) }\end{array}$ & $\begin{array}{l}\text { I'IR : } \mathrm{e} 32354 \\
\text { FIR : } \mathrm{b} 40767 \\
\text { PIR : d41080 }\end{array}$ & $\begin{array}{l}599 \\
221 \\
195\end{array}$ \\
\hline$B 65 D$ & $\begin{array}{l}\text { Phosphate regulon regulatory protein } \mathrm{PhoB} \\
\text { (Shigella dysenteriae) } \\
\text { (Escherichia coli) } \\
\text { Transcriptional regulatory protein OmpR } \\
\text { (Escherichia coli) }\end{array}$ & $\begin{array}{l}\text { PIR: a } 44753 \\
\text { PIR : rgecfb } \\
\text { PIR:a25024 }\end{array}$ & $\begin{array}{l}383 \\
382 \\
351\end{array}$ \\
\hline$B 65 E$ & $\begin{array}{l}\text { Sensor protein } \mathrm{BvgC} \\
\text { (Bortella pertussis) } \\
\text { ChvG protein } \\
\text { (Agrobacterium tumefaciens) }\end{array}$ & $\begin{array}{l}\text { SI':BVGC_BORPE } \\
\text { GP:ATUCHVG_1 }\end{array}$ & $\begin{array}{l}178 \\
169\end{array}$ \\
\hline$B 65 F$ & $\begin{array}{l}\text { Cell division protein FtsE } \\
\text { (Eschericbia coli) } \\
\text { ABC protein } \\
\text { (Escherichia coli) } \\
\text { Sulfate permease CysA } \\
\text { (Synechococcus sp.) }\end{array}$ & $\begin{array}{l}\text { PIR : ceecfe } \\
\text { SP:ABC_ECOLI }\end{array}$ & 375 \\
\hline
\end{tabular}

* ORFs which possess known proteins exhibiting significant homology (FASTA optimized score $>175$ ) are listed.

† Database entries of GP are followed by an underlined space and an ordinal number for each of the coding sequences. 
(a)

$\begin{array}{llllllllll}3190 & 3200 & 3210 & 3220 & 3230 & 3240 & 3250 & 3260 & 3270 & 3280\end{array}$ TGGGTAAGAAATTCGCTAACAACTGCGCAGGCGCTGAT TAAATTCTTAAACCAGCAGTACATCCATGTAGATGGAAGGAAGAGCCTTTTGTCGAAGG $-35-10$

Promoter-like sequence

$\begin{array}{lllllllll}3290 & 3300 & 3310 & 3320 & 3330 & 3340 & 3350 & 3360 & 3370\end{array}$ GATCTTCACGATTTTCGTCATGGAAACTATTAGGGATCGGGAGGCGCTTGAGCAGGATGCAGGCCATTTGAAAGTCTATCAAGGGAAAATGAACAA

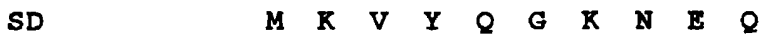

(b)

\section{B65C}

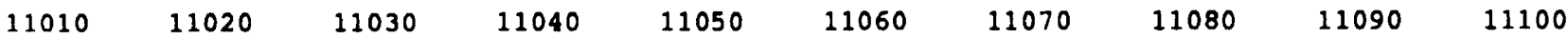
ATTTGACACCCGCCATTGAAGCCGTGGAGAGACAGTGCGAGCAAAATGAGAGAGTTCGGATCAGCCGgTAAAGCAGCTAAGCAGCAGGTCGGCTAAAG

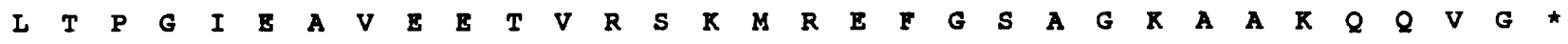

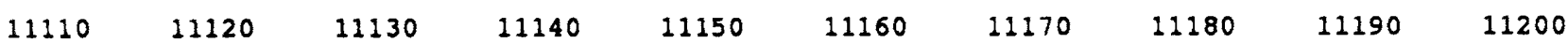
AGACCAGMAACCCCGAGATGGCGTGTPTTTTGTGCGGTTTGCGTCCATACGCCCAAAACGATTATGATGATGGCAGCAGTGTAAGAAACGGAGTGG Terminator-like sequence

SD

B65D

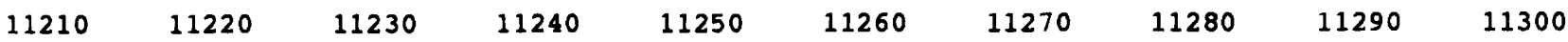
TTCAGTTTGAATAMATCATGATTGTGGAAGACAGTGAGACATTCGCGGATATTGCAGAATTACCTTGAAAAATACGGATATCAAACAGTGGTCGCCG $\begin{array}{lllllllllllllllllllllllllllllllll}M & N & K & I & M & I & V & E & D & S & E & D & I & R & G & L & L & Q & N & Y & I & E & K & Y & G & Y & Q & T & V & V & A & A\end{array}$ $\begin{array}{llllllllll}11310 & 11320 & 11330 & 11340 & 11350 & 11360 & 11370 & 11380 & 11390 & 11400\end{array}$ CGGATTTACAGCTGTTCTTGATGTCTTTTTGCGGAAAACCCGATGTGGTGCTGCTTGATATCAATTTGCGGCATATGACGGATATTATTGGTGCCG

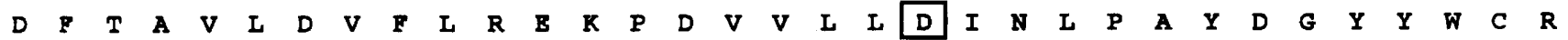

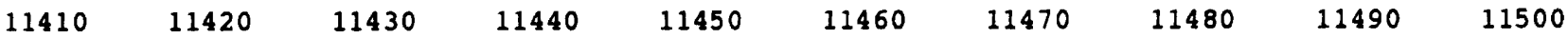
GCAGATCCGCCAGCACTCCACAAGCCCGATCATCTTTATTTCTGCCAGAAGCGGGGAAATGGATCAGGTGATGGCGATTGAAAATGGGGGAGACGATTAT

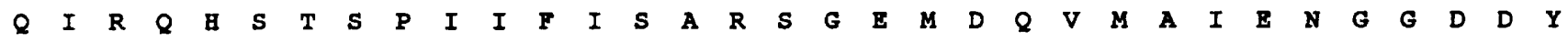

$\begin{array}{llllllllll}11510 & 11520 & 11530 & 11540 & 11550 & 11560 & 11570 & 11580 & 11590 & 11600\end{array}$ ATCGAAAAACCTTTTCTTATGATATTGTGCTTGCGAAAATCAAAAGCCAGATCCGGAGGGCGTACGGGGAGTACGCCGCAAAGCAGGGAGAGAAAGTGG $\begin{array}{lllllllllllllllllllllllllllllllllll}I & E & R & P & F & S & Y & D & I & V & L & A & K & I & K & S & Q & I & R & R & A & Y & G & E & Y & A & A & K & Q & G & E & K & V & V\end{array}$

$\begin{array}{llllllllll}11610 & 11620 & 11630 & 11640 & 11650 & 11660 & 11670 & 11680 & 11690 & 11700\end{array}$ TTGAATATGCCGCCGTTCAGCTCTTTGTGGAACGTTTGAACTGCGTTTTCAGGATGAAAAAATGAGCTTTCTAAAAAAGAAAGCAAGCTTTTGGAAGT

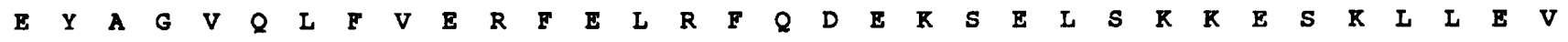

$\begin{array}{llllllllll}11710 & 11720 & 11730 & 11740 & 11750 & 11760 & 11770 & 11780 & 11790 & 11800\end{array}$ GCTECTTGAGCGGGGAGAAAGGTGACGAGTCGGGACCGTCTCATGGAAAAGACTGGGACACCGACATATTCATCGATGATAATACACTTAACGTGTAT $\begin{array}{lllllllllllllllllllllllllllllllllll}\mathbf{L} & \mathbf{L} & \mathbf{E} & \mathbf{R} & \mathbf{G} & \mathbf{E} & \mathbf{K} & \boldsymbol{V} & \mathbf{T} & \mathbf{S} & \mathbf{R} & \mathbf{D} & \mathbf{R} & \mathbf{L} & \mathbf{M} & \mathbf{E} & \mathbf{R} & \mathbf{T} & \mathbf{W} & \mathbf{D} & \mathbf{T} & \mathbf{D} & \mathbf{I} & \mathbf{F} & \mathbf{I} & \mathbf{D} & \mathbf{D} & \mathbf{N} & \mathbf{T} & \mathbf{L} & \mathbf{N} & \mathbf{V} & \mathbf{Y}\end{array}$

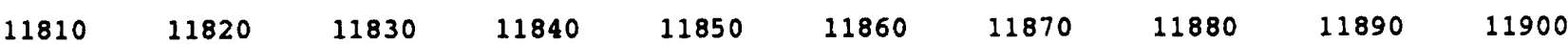
ATCACGCGGCTCAGAAAAAACTGCGGGAGCTGAATGCGCCTGTTTCTATTGAAGCGGTGCGGGGCGAAGGCTACCAGCTGAGGGCGCAGTCATGAAGCT

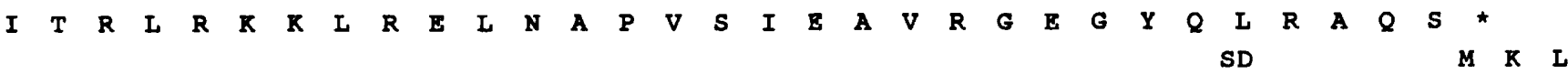

Fig. 2. For legend see p. 2295. 


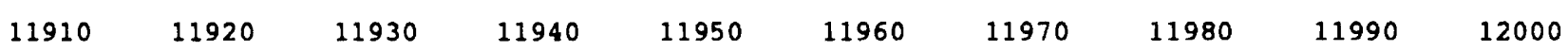
GTTCTCCGGTCTCATGCAGTGCTGATTCTGCTTTTTCTCCTTCAAGGGCTGTTTGTCTTCTTTTATTACTGGTTTGCGGGTCTCATTCTTTTCTCAC

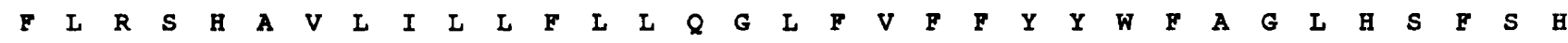

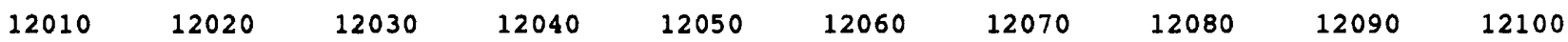
TTGTTTACATATTGGCGTGCAGCTTCTTATTTTGCGGGCTATCTTGCCTATCGCTGGTACAAAGACCGCGCGTGTATCATTGGCTCAGCTCGGGGC

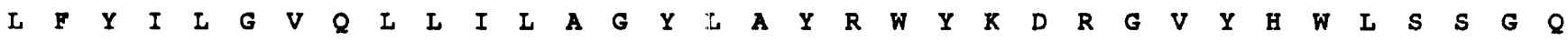

$\begin{array}{llllllllll}12110 & 12120 & 12130 & 12140 & 22150 & 12160 & 12170 & 12180 & 12190 & 12200\end{array}$ AGGAAGGGACGATATCCCGTATCTCGGTTCTTCAGTGTTCTGTT:AGAGCTGTATGAAAAGCAGATGGAGCTGATCCGGCTGCAGCACCAGAAGCTCCA

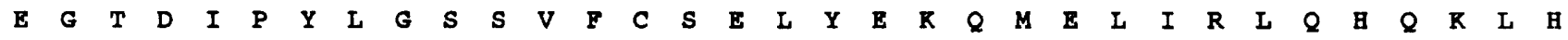

$\begin{array}{llllllllll}12210 & 12220 & 12230 & 12240 & 12250 & 12260 & 12270 & 12280 & 12290 & 12300\end{array}$ TGAGACCGAGCCAAGTTGACGCGCGGGTGACGTATATGATCAATGGGTGCACCAAGTAAAGACGCCGCTGTCGGTCATTAATTTAATCATTCAAGAA

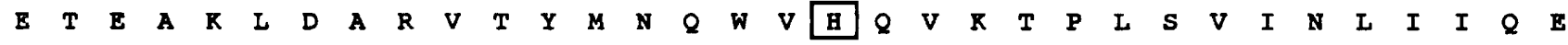

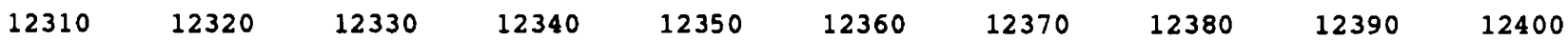
GAGGATGAGCCCGTTTTGAACAAATCAAAAAAGAAGTCCGGCAGATIGAATCGGGCTGGAGACGCTTCTTTATTCTTCAAGGCTCGACCTGTTTGAAC

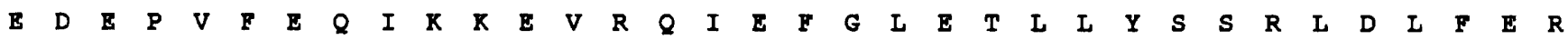

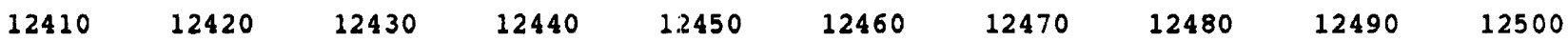
GGGATTTTAAAATCGAAGCGGTTCCCTGTCCGAGCTYCTCCAATCAGTTATTCAAAGCTATAAACGATTTTTTATTCAATATCGCGTTTATCCGAAAAT

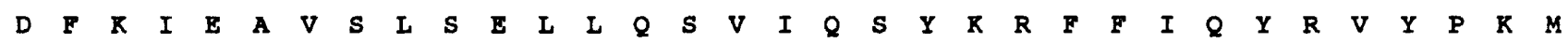

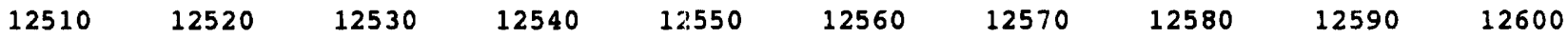
GAACGTTTGCGATGATCATCAGATTTACACCGATGCCAAATGGTCAAATTTGCGATTGGCCAAGTGGTCACAAATGCGGTCAAGTATTCAGCGGGCAAG

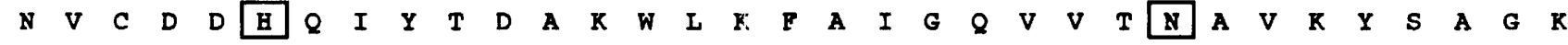

$\begin{array}{llllllllll}12610 & 12620 & 12630 & 12640 & 12650 & 12660 & 12670 & 12680 & 12690 & 12700\end{array}$ AGTGACAGACTTGAGCTGAATGTCTTCTGCGTGAAGACAGGACGGTGCTTGAGTCAAAGATTACGGTGTCGGGATTCCCTCACAAGACATCAAGCGGG

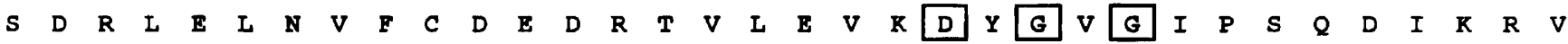

$\begin{array}{llllllllll}12710 & 12720 & 12730 & 12740 & 12750 & 12760 & 12770 & 12780 & 12790 & 12800\end{array}$ TGTTTGACCCTTATTACACGGTGAAACGGGCGGGTTTCCAAGAATCTACGGGATCGGCCTCCATCTCGTCAAAGAAATACAGATAAGCTCAATCA

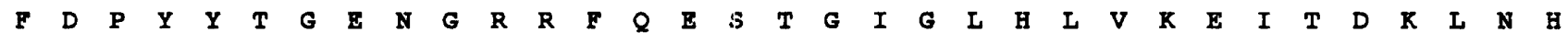

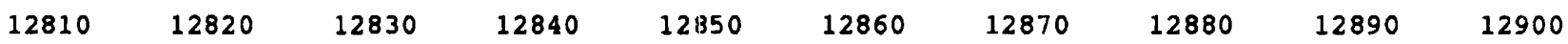
TACGGTGGACATCAGCTCGTCTCCCGGTAAGGGCATCAGTTCGATPTCATTTCTTACAAAAATGTAAGGCTGCGTTAAGAAACAACATAGCCCGCT

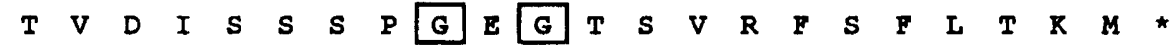

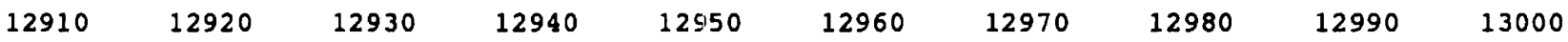
TTGTTTCCCCTTGATAACATGGATTTATGTCAAGGGGATTTTTATGTTGAACGTCCCGGCACTACATGAAAGCAGCCCAGATCAACAAGAATAAACCAT Terminator-like sequence

\section{B65F}

$\begin{array}{llllllllll}13010 & 13020 & 13030 & 13040 & 13050 & 13060 & 13070 & 13080 & 13090 & 13100\end{array}$ AAGGAGGATTTYCATGGCGATATGCTTGAAGTCAACATATAAATAAAACTATAAAGGACAAGTGTCCTATCAGGCCTTAAAGCAAATTCATTTTCA

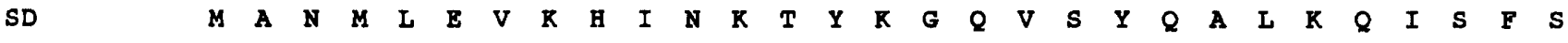

$\begin{array}{lllllllll}13110 & 13120 & 13130 & 13140 & 13150 & 13160 & 13170 & 13180 & 13190\end{array}$ ATAGAAGAAGCGAGTTCACGCCGGTGATGGGGCCGTCCGGGTCCGGAAAACAACGCTTTTGAATATCATTTCAACGATTGACCGTCCAGATTCAGGCG

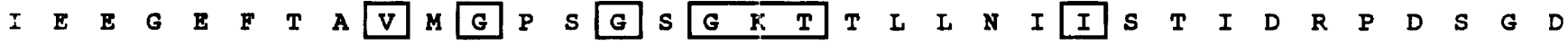
ATP-binding cassette

Fig. 2. For legend see facing page. 
Nucleotide sequence of the B. subtilis iol region

$\begin{array}{llllllllll}13210 & 13220 & 13230 & 13240 & 13250 & 13260 & 13270 & 13280 & 13290 & 13300\end{array}$ ATATCCTGATTAACGGAGAAAACCCCATCGCCTGAAGCGGACAAAGCTCGCTCATTTCCGCCGCAAGCAGCTCGGATTTGTGTTTCAGGATTTCAACCT

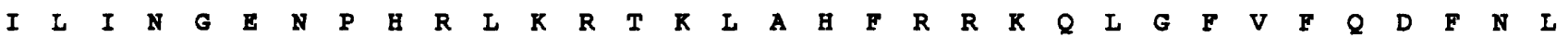

$\begin{array}{llllllllll}13310 & 13320 & 13330 & 13340 & 13350 & 13360 & 13370 & 13380 & 13390 & 13400\end{array}$

TCTGGATACGCTGACGATCGTGAAATATCATGCTGCCGTTAACGCTTGAAAAAGAAGCGCCGTCTGTAATGGAAGAAAAACTGCACGGCATCGCGGCA

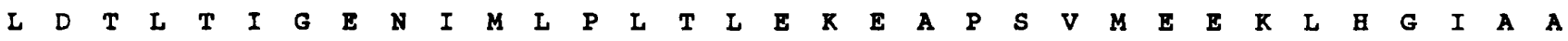

$\begin{array}{llllllllll}13410 & 13420 & 13430 & 13440 & 13450 & 13460 & 13470 & 13480 & 13490 & 13500\end{array}$

AAGCTCGGTATCGAAAACCTGCTTAACAAGCGGACGTTTGAATTATCCGAGGCCAGCGCCAGCGGGCCGCCATCGCAAGAGCGGTCATTCATAAGCCGT

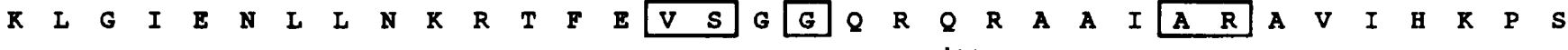
Transmitter

$\begin{array}{llllllllll}13510 & 13520 & 13530 & 13540 & 13550 & 13560 & 13570 & 13580 & 13590 & 13600\end{array}$ CACTCATTCTGGCCGATGAACCAACTGGAACCTTGATTCCAAGCGACAAAGACGTCATGGAAACGTGCAGAGCCTGATCGGGATGATCATGTCAC

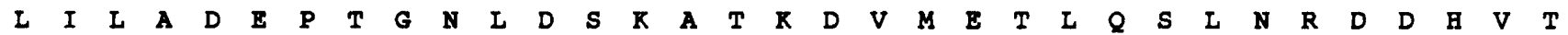

$\begin{array}{llllllllll}13610 & 13620 & 13630 & 13640 & 13650 & 13660 & 13670 & 13680 & 13690 & 13700\end{array}$ GGCTTTGATGGTGACCCACGATCCGGTTTCGGCAAGCTACTGCCGCCGCGTCATCTTTATAAAAGACGTCGTACTGTTTAATGAGATTTACCGCGGGGAA

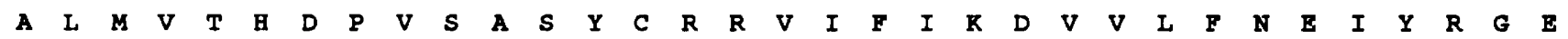
B65G

$\begin{array}{llllllllll}13710 & 13720 & 13730 & 13740 & 13750 & 13760 & 13770 & 13780 & 13790 & 13800\end{array}$
AACCGCCAATATITTATGACAATTCTCGATGTGCTGTCTATGCTGGGGGGAACGCAAATACCTTTCTTCAGTTCGCTTATAAAAACGTCACGAGA $\begin{array}{llllllllllllllllllllllllllllll}N & R & Q & V & F & Y & E & Q & I & L & D & V & L & S & M & L & G & G & \text { N } & A & N & D & I & S & S & V & R & L & \text { * }\end{array}$ $\begin{array}{lllllllllllllll}\text { SD } & \text { M } & T & \mathbf{F} & \text { L } & Q & \mathbf{F} & \mathbf{A} & \mathbf{Y} & \mathrm{K} & \mathbf{N} & \boldsymbol{V} & \mathbf{T} & \mathbf{R}\end{array}$

Fig. 2. Specific features found in the nucleotide sequence of the iol region. Two sequence segments derived from the sequence ( 1 to $14974 \mathrm{nt}$ ) obtained by sequencing both strands independently are presented; (a) is that of the region upstream of the E83D gene ( 3181 to $3380 \mathrm{nt}$ ) and (b) is that containing the B65C (partial ORF, 11001 to $11095 \mathrm{nt}$ ), B65D (11207 to $11893 \mathrm{nt}$ ), B65E (11893 to $12867 \mathrm{nt}$ ), B65F (13014 to $13784 \mathrm{nt}$ ), and B65G (partial ORF, 13762 to $13800 \mathrm{nt}$ ) genes. The SD sequences for these ORFs are underlined and labelled SD. The aa sequences of their products are shown as single letters with the respective termination codons indicated by asterisks. The ' $-35^{\prime}$ ' (3198 to $3203 \mathrm{nt}$ ) and ' $-10^{\prime}$ ' (3221 to $3226 \mathrm{nt}$ ) regions of the putative promoter-like sequence for the iol operon are located upstream of the E83D gene (doubly underlined). Two palindromic sequences within the respective $\rho$-independent terminator-like sequences (11108 to 11129 and 12906 to $12938 \mathrm{nt}$ ) are indicated by underlining; one might be a transcription terminator for the putative iol operon, which is located downstream of the $B 65 C$ gene, and the other might be that for the B65D and B65E genes comprising the two-component regulatory system, which is located between the $B 65 E$ and $B 65 F$ genes. The two aspartate $(D)$ and one lysine $(K)$ residues boxed in the aa sequence of the B65D protein are those highly conserved in most response regulators of the two-component regulatory systems of bacteria (Stock et al., 1989). The boxed residues in the aa sequence of the B65E protein are the highly-conserved residues among histidine protein kinases of the two-component regulatory systems (Stock et al., 1989). In the aa sequence of the B65F protein, the residues in the ATP-binding domain are boxed and labelled as the 'ATP-binding cassette' (Higgins et al., 1990); those of another conserved region are boxed and labelled as the 'Transmitter', proposed to be involved in the transmission of an ATP-dependent conformational change to the membrane-associated sites (Gill et al., 1986).

gene is located within the $30 \mathrm{~N}$ fragment between 4029 and $4082 \mathrm{~kb}$ (M. Itaya, personal communication). The locations of the $2.65 \mathrm{~kb}$ HindIII fragment and the HindIII insert in plasmid pNEXT40 were determined by analysing their Southern hybridization patterns using restriction enzyme fragments of the inserts of these recombinant clones (data not shown). With reference to the physical map of the B. subtilis chromosome (Itaya \& Tanaka, 1991), these results clearly indicate that the direction of ori $C$ is from the $i d b$ gene to this Not I site, as shown for the direction of gnt, sacS and the movement of the replication fork (Fig. 1).

The nucleotide sequence of the $1.6 \mathrm{~kb}$ region containing the $i d h$ gene has been reported previously (Fujita et al., 1991). To extend the sequences of the adjacent regions to the $i d h$ gene, an $8.3 \mathrm{~kb}$ EcoRI fragment (E83) of the EM11 clone and a $6.5 \mathrm{~kb} \mathrm{BamHI}$ fragment (B65) of the EM14 clone (Fig. 1), which are located upstream and downstream of the $i d b$ gene, respectively, were isolated and sequenced systematically. Connection of the nucleotide sequences of the $8.3 \mathrm{~kb}$ and $6.5 \mathrm{~kb}$ fragments newly determined and the $1.6 \mathrm{~kb}$ region resulted in a $14974 \mathrm{bp}$ nucleotide sequence for the region containing the $i d h$ gene. Analysis of the $14974 \mathrm{bp}$ sequence revealed 12 complete open reading frames (ORFs; genes) [E83B, E83C, E83D, E83E, E83F, E83G (idh), B65A, B65B, $B 65 C, B 65 D, B 65 E$ and $B 65 F]$ and two partial ones 
(E83A and B65G) (Fig. 1). The endpoints of these genes in the sequence and their features, such as the molecular size and the putative SD sequence and initiation codon, are shown in Table 1 . (The $B 65 F$ gene overlaps the $B 65 C^{\circ}$ gene by 23 bp.)

Eleven out of the 13 genes, except the first partial ORF$E 83 \mathrm{~A}$, were predicted to use ATG as initiation codon, though the E83D and B65D genes might use TTG, suggesting that the genes in the iol region might utilize ATG more preferentially than those in other region; (personal communications from other Japanese group's participating in the B. subtilis genomic sequencing project; Glaser et al., 1993). More interesting is that the direction of the transcription of all these genes is the same as that of the movement of the replication fork. This is often observed in regions located relatively close to the chromosome origin like the iol region (Zeigler \& Dean, 1990; Glaser et al., 1993).

\section{Functions of the newly identified genes}

Among the putative gene products encoded by the 12 complete ORFs, only the product of the $E 83 G$ gene was previously identified as the $i d h$ gene encoding inositol dehydrogenase (Fujita et al., 1991). As a prerequisite for determining the functions of these putative gene products, we searched for homology with known protein sequences present in sequence libraries, using the FASTA program to obtain the FASTA optimized score (Pearson \& Lipman, 1988). (The last homology search was performed in November 1993.) Eight out of the 12 gene products exhibited significant homology to known proteins in the PIR, SP and GP Protein Databases (FASTA optimized score > 175) (Table 2). However, four products (E83B, E83E, B65A and B65B) seemed to be unique, exhibiting no significant homology to known proteins (FASTA optimized score $<100$ ).

We discuss below the functions of the putative gene products exhibiting significant homology to known proteins. The E83C protein showed homology to various fructokinases including the Solanum tuberosum L. enzyme (Smith et al., 1993), implying that this protein might be a fructokinase. The E83D protein showed homology to many acetolactate synthases, including a yeast enzyme (Falco et al., 1985), acetohydroxy acid synthase, and the pyruvate dehydrogenase of $E$. coli, implying that this protein might have a functional relationship with these enzymes. The E83F protein exhibited homology to the methylenomycin A resistant protein and the NorA protein of Staphylococcus aureus participating in the active efflux pump for hydrophilic quinolones (Yoshida et al., 1990), and to D1 and arabinose transporter proteins. This suggests that it might be involved in a membraneassociated transport system. The gene product of E83C; $(i d h)$, which was identified as inositol dehydrogenase of $B$. subtilis (Fujita et al., 1991), showed high homology to the StrI protein of Streptomyces griseus, which was predicted to catalyse one of the numerous steps dependent on dehydrogenases or epimerases in the biosynthesis of hexose- derived moieties of streptomycin (Mansouri \& Piepersberg, 1991). The B65C protein exhibited homology to many fructose-bisphosphate aldolases including the Rhodobacter sphaeroides enzyme (Gibson et al., 1991), implying that this protein might have a similar function to this enzyme. Moreover, this protein exhibited very high homology to the Tst protein of B. subtilis, which is required for RNA synthesis (Trach et al., 1988). Recently, Mitchell et al. (1992) found that this $t s r$ gene was a portion of the fla1 gene encoding the fructose-1,6-bisphosphate aldolase. Moreover, Sukhodolets et al. (1983) reported that the B. subtilis dra gene encoding deoxyriboaldolase was located at $339^{\circ}$, which is very close to the $B 65 C$ gene. It remains to be examined whether or not the $d r a$ gene is the $B 65 C$ gene.

The $\mathrm{B} 65 \mathrm{D}$ protein showed high homology to many proteins belonging to the OmpR subfamily of response regulator proteins of the two-component regulatory systems of bacteria (Stock et al., 1989), such as PhoB proteins including that of E. coli (Makino et al., 1986), and the OmpR protein of E. coli (Comeau et al., 1985). When the sequence of this protein was aligned with those of these proteins, two aspartate residues and a lysine residue highly conserved in most response regulators were found in it, as shown in Fig. 2. These two aspartate residues are considered to be phosphorylated by the phosphohistidine of the corresponding histidine protein kinase (Stock et al., 1989). Moreover, the B65E protein exhibited homology to histidine protein kinases of the two-component regulatory systems (Stock et al., 1989) including the sensor protein BvgC of Bordetella pertussis (Arico et al., 1989). Alignment of the sequence of this protein with those of these histidine protein kinases revealed histidine residues in $\mathrm{B} 65 \mathrm{E}$ which might be phosphorylated and aa residues conserved in these histidine kinases (Fig. 2). Interestingly, the $B 65 D$ gene is adjacent to $B 65 E$, so their gene products very likely constitute a set of the two-component regulatory system (a response regulator protein and its histidine protein kinase). The function of this set remains to be determined.

The $\mathrm{B} 65 \mathrm{~F}$ protein showed very high homology to many proteins belonging to the $\mathrm{ABC}$ family (Higgins et al., $1990)$, such as the $A B C$ protein of $E$. coli, the sulfate permease of Synechococcus sp. and the FtsE protein of $E$. coli (Gill et al., 1986), suggesting that it might function as an ATP-dependent transporter for an unidentified substrate. A domain of the $\mathrm{B} 65 \mathrm{~F}$ protein for the ATP-binding cassette was predicted by alignment of the sequence of this protein with those of transporter proteins belonging to the $\mathrm{ABC}$ family, as shown in Fig. 2 (Higgins et al., 1990). Fig. 2 also shows another conserved domain of this family of proteins, which was recently proposed to be involved in transmission of an ATP-dependent conformational change to membrane-associated sites (Gibbs $e t$ al., 1992). The $B 65 F$ gene is located immediately downstream of the $B 65 D$ and $B 65 E$ genes comprising the twocomponent regulatory system, although a stem-loop structure which might possibly function as transcription terminator exists between the B65E and B65F genes (Fig. 
2). It would be very interesting to elucidate the actual functions of these three genes and their mutual relation.

\section{Structure of the iol operon}

The $15 \mathrm{~kb}$ region, the nucleotide sequence of which was determined, is believed to contain the ioloperon, involved in myo-inositol catabolism in B. subtilis, because not only the $i d h$ gene encoding inositol dehydrogenase but also many iol mutations rendering the cells unable to grow on myo-inositol are located in this region (Y. Fujita, unpublished observation). Although the pathway for myoinositol degradation is not well studied in B. subtilis, an outline of this pathway in Aerobacter aerogenes (Klebsiella pneumoniae) has been elucidated (Berman \& Magasanik, 1966; Anderson \& Magasanik, 1971). myo-Inositol transported into cells is dehydrogenated by inositol dehydrogenase. The resulting 2-keto-myo-inositol is dehydrated to D-2,3,-diketo-4-deoxy-epi-inositol, which is then hydrated to 2-deoxy-5-keto-D-gluconic acid, the first open-chain intermediate. After this intermediate has been phosphorylated by a kinase, an aldolase catalyses the cleavage of the kinase product (2-deoxy-5-keto-D-gluconic acid 6-phosphate) to yield dihydroxyacetone phosphate and malonic semialdehyde. The malonic semialdehyde is metabolized by malonic semialdehyde oxidative decarboxylase, which catalyses the conversion of malonic semialdehyde, coenzyme $\mathrm{A}$ and $\mathrm{NAD}^{+}$to acetyl-CoA, $\mathrm{CO}_{2}$ and $\mathrm{NADH}+\mathrm{H}^{+}$.

To identify the genes of the iol operon, we first searched for promoter-like sequences recognized by the major $\sigma^{\mathrm{A}}$ factor of B. subtilis using a personal computer, and found in the $14974 \mathrm{bp}$ sequence a plausible promoter-like sequence only immediately upstream of the E83D gene, the ' -35 ' and ' -10 ' regions of which are shown in Fig. 2. We also looked for a $\rho$-independent terminator which might be located downstream of the E83G (idh) gene encoding inositol dehydrogenase, and found a terminatorlike palindromic sequence followed by a stretch of $T$ residues downstream of the $B 65 C$ gene, the location of which is shown in Fig. 2. These results also imply that the $B 65 D$ and $B 65 E$ genes might be transcribed by RNA polymerase associated with another $\sigma$ factor. Thus, it was considered, on the basis of the location of $i d h$, and the above transcriptional promoter- and terminator-like sequences, that the iol operon might consist of seven genes (E83D, E83E, E83F, idh, B65A, B65B and B65C). Moreover, assuming that the pathway for myo-inositol degradation in B. subtilis may be similar to that in Aerobacter aerogenes, we could hypothesize as to the functions of some of these genes from the results of the homology search for their products. The third protein, E83F, showed homology to various membrane-associated transporter proteins, as described above, which suggests that it might be involved in the transport of myo-inositol. The transported myo-inositol could be dehydrogenated by the inositol dehydrogenase encoded by $i d h$. The resulting open-chain phosphorylated compound, 2-deoxy-5-ketoD-gluconic acid 6-phosphate, might be cleaved by the $\mathrm{B} 65 \mathrm{C}$ protein, which showed homology to various aldolases. The resulting malonic semialdehyde might be finally metabolized to acetyl-CoA, $\mathrm{CO}_{2}$ and $\mathrm{NADH}+\mathrm{H}^{+}$ by the E83D protein, because this protein showed homology not only to various acetolactate synthases but also to pyruvate dehydrogenase, an enzyme similar to malonic semialdehyde oxidative decarboxylase.

The expression of the $i d h$ gene is induced by the addition of myo-inositol to the medium (Fujita \& Freese, 1981), suggesting that expression of the iol operon may be induced by myo-inositol. Thus, there should be a transcriptional regulator for the iol operon. However, no gene encoding a protein showing homology to DNA-binding regulatory proteins was found within the putative iol operon, suggesting that the gene for the transcriptional regulator should be located elsewhere. Experimental analysis of the organization and transcription of the iol operon is in progress.

\section{ACKNOWLEDGEMENTS}

The authors wish to thank the following undergraduate students, T. Tokuyama and T. Watanabe, for their help with the experiments. This work was supported by a Grant-in-Aid for Creative Basic Research on 'Human Genome Analysis' from the Ministry of Education, Science and Culture of Japan.

\section{REFERENCES}

Anderson, W. A. \& Magasanik, B. (1971). The pathway of myoinositol degradation in Aerobacter aerogenes. Conversion of 2-deoxy5-keto-D-gluconic acid to glycolytic intermediates. $J$ Biol Chem $\mathbf{2 4 6}$, 5662-5675.

Arico, B., Miller, J. F., Roy, C., Stibitz, S., Monack, D., Falkow, S., Gross, R. \& Rappouli, R. (1989). Sequences required for expression of Bordetella pertussis virulence factors share homology with prokaryotic signal transduction proteins. Proc Natl Acad Sci US $A$ 86, 6671-6675.

Benton, W. D. \& Davis, R. W. (1977). Screening $\lambda$ gt recombinant clones by hybridization to single plaques in situ. Science 196, 180-182.

Berman, T. \& Magasanik, B. (1966). The pathway of myo-inositol degradation in Aerobacter aerogenes. Dehydrogenation and dehydration. J Biol Chem 241, 800-806.

Comeau, D. E., Ikenaka, K., Tsung, K. \& Inouye, M. (1985). Primary characterization of the protein products of the Escherichia coli omp $B$ locus: structure and regulation of synthesis of the $\mathrm{OmpR}$ and EnvZ proteins. $J$ Bacteriol 164, 578-584.

Falco, S. C., Dumas, K. S. \& Livak, K. J. (1985). Nucleotide sequence of the yeast $I L V 2$ gene which encodes acetolactate synthase. Nucleic Acids Res 13, 4011-4027.

Fujita, Y. \& Freese, E. (1981). Isolation and properties of a Bacillus subtilis mutant unable to produce fructose-bisphosphatase. $J$ Bacteriol 145, 760-767.

Fujita, Y. \& Fujita, T. (1983). Genetic analysis of a pleiotropic deletion mutant $(\Delta i g f)$ in Bacillus subtilis. J Bacteriol 154, 864-869.

Fujita, Y., Shindo, K., Miwa, Y. \& Yoshida, K. (1991). Bacillus subtilis inositol dehydrogenase-encoding gene $(i d h)$ : sequence and expression in Escherichia coli. Gene 108, 121-125.

Gibbs, T. W., Gill, D. R. \& Salmond, G. P. C. (1992). Localized mutagenesis of the fts $Y E X$ operon: conditionally lethal missense substitutions in the FtsE cell division protein of Eschericbia coli are 
similar to those found in the cystic fibrosis transmembrane conductance regulator protein (CFTR) of human patients. Mol \& Gen Genet 234, 121-128.

Gibson, J. L., Falcone, D. L. \& Tabita, F. R. (1991). Nucleotide sequence, transcriptional analysis, and expression of genes encoded within the form $\mathrm{I} \mathrm{CO}_{2}$ fixation operon of Rhodobacter sphaeroides. $J$ Biol Chem 266, 14646-14653.

Gill, D. R., Hatfull, G. F. \& Salmond, G. P. C. (1986). A new cell division operon in Escherichia coli. Mol \& Gen Genet 205, 134-145.

Glaser, P., Kunst, F., Arnaud, M., Coudart, M.-P., Gonzales, W., Hullo, M.-F., Ionescu, M., Lubochinsky, B., Marcelino, L., Moszer, I., Presecan, E., Santana, M., Schneider, E., Schweizer, J., Vertes, A., Rapoport, G. \& Danchin, A. (1993). Bacillus subtilis genome project : cloning and sequencing of the $97 \mathrm{~kb}$ region from $325^{\circ}$ to $3.33^{\circ}$. Mol Microbiol 10, 371-384.

Higgins, C. F., Hyde, S. C., Mimmack, M. M., Gileadi, U., Gill, D. R. \& Gallagher, M. P. (1990). Binding protein-dependent transport systems. J Bioenerg Biomembr 22, 571-592.

Itaya, M. \& Tanaka, T. (1991). Complete physical map of the Bacillus subtilis 168 chromosome constructed by a gene-directed mutagenesis method. $J$ Mol Biol 220, 631-648.

Kohara, Y., Akiyama, K. \& Isono, K. (1987). The physical map of the whole E. coli chromosome: application of a new strategy for rapid analysis and sorting of a large genomic library. Cell 50, 495-508.

Makino, K., Shinagawa, H., Amemura, M. \& Nakata, A. (1986). Nucleotide sequence of the $p h o B$ gene, the positive regulatory gene for the phosphate regulon of Eschericbia coli. J Mol Biol 190, 37-44.

Mansouri, K. \& Piepersberg, W. (1991). Genetics of streptomycin production in Streptomyces griseus: nucleotide sequence of five geres,

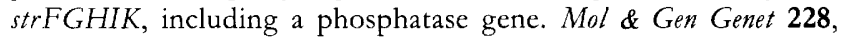
$459-469$.
Mitchell, C., Morris, P. W., Lum, L., Spiegelman, G. \& Vary, J. C. (1992). The amino acid sequence of a Bacillus subtilis phosphoprotein that matches an orf $Y$-tsr coding sequence. Mol Microbiol 6, 1345-1349.

Pearson, W. R. \& Lipman, D. J. (1988). Improved tools for biological sequence comparison. Proc Natl Acad Sci USA 85, 2444-2448.

Smith, S. B., Taylor, M. A., Burch, L. R. \& Davies, H. V. (1993). Primary structure and characterization of a cDNA clone of fructokinase from potato (Solanum tuberosum L. CV Record). Plant Pbysiol 102, 1043.

Southern, E. M. (1975). Detection of specific sequences among DNA fragments separated by gel electrophoresis. J Mol Biol 98, 503-517.

Stock, J. B., Ninfa, A. J. \& Stock, A. M. (1989). Protein phosphorylation and regulation of adaptive responses in bacteria. Microbiol Rev 53, 450-490.

Sukhodolets, V. V., Flyakh, Y. V. \& Rumyantseva, E. V. (1983). Mapping of mutations in genes for nucleoside catabolism on the Bacillus subtilis chromosome. Genetika 19, 221-226.

Trach, K., Chapman, J. W., Piggot, P., Lecoq, D. \& Hoch, J. A. (1988). Complete sequence and transcriptional analysis of the spoOF region of the Bacillus subtilis chromosome. $J$ Bacteriol 170, 4194-4208.

Yoshida, H., Bogaki, M., Nakamura, S., Ubukata, K. \& Konno, M. (1990). Nucleotide sequence and characterization of the Stapbylococcus aureus nor $A$ gene, which confers resistance to quinolones. $J$ Bacteriol 172, 6942-6949.

Zeigler, D. R. \& Dean, D. H. (1990). Orientation of genes in the Bacillus subtilis chromosome. Genetics 125, 703-708.

Received 5 January 1994; revised 19 March 1994; accepted 15 April 1994. 